\title{
Predicting Stock Prices Using Polynomial Classifiers: The Case of Dubai Financial Market
}

\author{
Khaled Assaleh ${ }^{1}$, Hazim El-Baz ${ }^{2}$, Saeed Al-Salkhadi ${ }^{2}$ \\ ${ }^{1}$ Department of Electrical Engineering, American University of Sharjah, Sharjah, UAE; ${ }^{2}$ Engineering Systems Management Graduate \\ Program, American University of Sharjah, Sharjah, UAE. \\ Email: kassaleh@aus.edu
}

Received July $10^{\text {th }}, 2010$; revised October $1^{\text {st }}, 2010$; accepted December $1^{\text {st }}, 2010$.

\begin{abstract}
Predicting stock price movements is a challenging task for academicians and practitioners. In particular, forecasting price movements in emerging markets seems to be more elusive because they are usually more volatile often accompanied by thin trading-volumes and they are susceptible to more manipulation compared to mature markets. Technical analysis of stocks and commodities has become a science on its own; quantitative methods and techniques have been applied by many practitioners to forecast price movements. Lagging and sometimes leading technical indicators provide rich quantitative tools for traders and investors in their attempt to gain advantage when making investment or trading decisions. Artificial Neural Networks (ANN) have been used widely in predicting stock prices because of their capability in capturing the non-linearity that often exists in price movements. Recently, Polynomial Classifiers (PC) have been applied to various recognition and classification application and showed favorable results in terms of recognition rates and computational complexity as compared to ANN. In this paper, we present two prediction models for predicting securities' prices. The first model was developed using back propagation feed forward neural networks. The second model was developed using polynomial classifiers $(P C)$, as a first time application for PC to be used in stock prices prediction. The inputs to both models were identical, and both models were trained and tested on the same data. The study was conducted on Dubai Financial Market as an emerging market and applied to two of the market's leading stocks. In general, both models achieved very good results in terms of mean absolute error percentage. Both models show an average error around $1.5 \%$ predicting the next day price, an average error of $2.5 \%$ when predicting second day price, and an average error of $4 \%$ when predicted the third day price.
\end{abstract}

Keywords: Dubai Financial Market, Polynomial Classifiers, Stock Market, Neural Networks

\section{Introduction}

Accurate prediction of stock prices presents a challenging task for traders and investors. Multitude of economical, social, political and psychological factors interacts in a complex way to form stock movement patterns. The early Efficient Market Theory (EMT) claims that prices move in a random way and it is not possible to develop an algorithm of any kind that predicts stock prices [1]. Other researchers contradicted this claim and presented considerable evidence showing that stock prices are, to some extent, predictable. Forecasting or predicting stock prices may be done following one or a combination of four approaches: fundamental analysis approach, technical analysis approach, time series forecasting and machine learning. Each approach has its own merits as well as limitations.
In recent years, ANN has been successfully used for modeling financial time series. For example, the back propagation ANN was used to forecast future price movements of Kuala Lumpur Stock Exchange which is major emerging market [2]. Brownstone [3] reported the prediction error of neural networks measured by the mean square error and the root mean square error to predict daily market close 5 days and 25 days ahead for the Financial Times Stock Exchange (FTSE) in the UK. Lee and Jo [4] developed an expert system using the Japanese Candlestick chart patterns to predict the proper timing of stocks and reported a success ratio of $72 \%$ of their system. Template matching, from pattern recognition, and the feed forward neural networks were combined and used to forecast stock market activity in the New York Stock Exchange Composite index [5]. Another learning system that integrates genetic algorithm (GA) and Sup- 
port Vector machines (SVM) for stock market prediction was reported by Choudhry and Garg [6]. The next day stock price of stock market indexes using a hybrid approach that integrates both GA and ANN was reported by Armano et al. [7]. In a different but related application, the NN was used to capture the underlying rules of movements in currency exchange rates with reasonable results in [8]. The use of Artificial Neural Networks to predict stock market behavior in terms of its direction was reported in [9] for Istanbul Stock Exchange where the historical data was grouped in seven different prediction systems models to which eight different ANN and Logistics Regressions models were applied. Chena et al. [10] used the probabilistic neural network (PNN) to forecast the direction of Taiwan Stock Marekt Index return using historical data. Enke and Thawornwong [11] combined information gain technique of data mining and Neural Network to evaluate the predictive relationship of financial and economic variables. Caoa et al. [12] compared financial forecasting linear models to the predictive power of univariate and multivariate Neural Networks for the Chinese stock market. Kaastra and Boyd [13] provided an eight-step procedure to design neural network forecasting model with trade-offs in parameters selection and common pitfalls. Kohzadi et al. [14] examined whether neural networks can outperform a traditional autoregressive integrated moving average (ARIMA) model for forecasting commodity prices. Olsona Mossmanb [15] compared the prediction accuracy of neural network and logistic regression technique using historical data of Canadian Stock exchange

Common to all the reviewed prediction methods is the use of a pattern classifier which in most cases is some sort of a neural classifier. Whether used to model the manifolds of each class or to discriminate the patterns of different classes, neural classifiers can be divided into relative density models and discriminative models. Examples of relative density models include linear mixture models and auto-association networks. Whereas, discriminative neural classifiers include the multi-layer perceptron (MLP), the radial basis function (RBF) networks, and the polynomial classifier (PC) [16].

PCs can be described as higher-order neural networks which consist of a single-layer network with the polynomial terms of patterns feature as inputs [16]. The polynomial classifiers are learning algorithms proposed and adopted in recent years for regression, classification and recognition with significant properties and generalization capability [16].

Due to their need for less training examples and far less computational requirements, PCs have shown superior performance to multilayer neural networks. Polynomial classifiers have been successfully used for various pattern recognition and signal detection applications including speech and speaker recognition $[17,18]$. Another recent study on the recognition of sign language alphabet using polynomial classifiers delivered superior recognition results [19] over Adaptive Neurofuzzy Inference systems (ANFIS). More applications of PCs have been reported in modeling of tool wear $[20,21]$ and noninvasive fetal ECG extraction.

This paper describes the use of the Polynomial Classifiers (PC) to predict stock prices in the Dubai Financial Market in the United Arab Emirates and compares the results with those obtained by using Artificial Neural Networks (ANN).

It is worth mentioning here that, at the time of this research, literature review revealed that there is no reported research that applied the polynomial classifiers for the prediction of stock price movements. In addition, there were no research found that reported using neural network to predict stock price movements in Dubai Financial Market.

\section{Dubai Financial Market (DFM)}

In the last years of the previous century, the United Arab Emirates (UAE) became one of the fastest developing countries in the Middle East and South Asia. Dubai, which is considered the commercial capital and the center of international business in the UAE, has taken the leadership in developing and modernizing both governmental and private sectors with state-of-the-art strategies, policies, technologies and infrastructure. One of the fastest and most powerful growing sectors in the UAE is finance.

On March $26^{\text {th }}, 2000$, Dubai Financial Market commenced operation with listing shares of seven companies and ten brokers. DFM has grown rapidly and has scored noticeable records in terms of trading volume and market values. At the time of this study, more than 50 companies are listed with the DFM and it is considered one of the leading financial markets in the Middle East.

Two of DFM's leading stocks were selected for this research; Emaar Properties (EMAAR) and Dubai Islamic Bank (DIB). EMAAR is the leading real estate developer in the Middle East and DIB the world's first fullyfledged Islamic bank. These stocks were chosen because they have sufficient historical data, actively traded, and each to represent different sectors in the UAE economy. The data used for both stocks is the closing prices covering the period of April 2000 to March 2006 (total of 2176 data points). These data points are the daily closing stock prices in the currency of the United Arab Emirates Dirham (AED). The AED is tied with the USD with a conversion rate of approximately $1 \mathrm{USD}=3.67$ AED. 


\section{Background of Modeling Techniques}

In this work we have used Artificial Neural Networks (ANN) and Polynomial Classifiers (PC) as modeling techniques to predict stock prices from historical price data. The ANN that we used is the standard feed forward architecture trained with the standard back propagation method. Since this architecture is widely used and very well known we felt that it would be redundant to explain it here. The reader can refer to [2] for more details about feed forward back propagation ANN. On the other hand polynomial classifier being used for the first time for this application is explained here with adequate details.

\subsection{Polynomial Classifiers}

Polynomial Classifiers (PCs) provide an efficient method for describing non-linear input/output relationships. To model a sequence of input/output data using polynomial classifiers, a set of parameters, also referred to as weights, are determined such that the multi-dimensional feature vectors, which represent the input sequence, are best mapped to the corresponding output sequence. For the problem at hand (i.e. stock price prediction) our input is a sequence of feature vectors formed from the historical prices of the stock being studied. For each of the input feature vectors the corresponding output is the future price of the stock.

Consequently, the training data is arranged into a $N$ $d$-dimensional feature vectors arranged in the $N \times d$ matrix $\boldsymbol{X}$, along with its corresponding $N$ dimensional column vector $\boldsymbol{t}_{\boldsymbol{x}}$ representing the target vector (future prices). For instance, to predict the next day price from the previous $d$ days we arrange the given training time series of dimension $N+d+1$ data as follows.

$$
\begin{gathered}
\boldsymbol{X}=\left[\begin{array}{c}
\boldsymbol{x}_{1} \\
\boldsymbol{x}_{2} \\
\vdots \\
\boldsymbol{x}_{N}
\end{array}\right]=\left[\begin{array}{cccc}
x_{1} & x_{2} & \cdots & x_{d} \\
x_{2} & x_{3} & \cdots & x_{d+1} \\
\vdots & \vdots & \ddots & \vdots \\
x_{N} & x_{N+1} & \cdots & x_{N+d}
\end{array}\right] \\
\boldsymbol{t}_{x}=\left[\begin{array}{c}
x_{d+1} \\
x_{d+2} \\
\vdots \\
x_{N+d+1}
\end{array}\right]
\end{gathered}
$$

A $K^{\text {th }}$ order polynomial classifier consists of $K^{\text {th }}$ order polynomial expansion of the d-dimensional feature vector, $\boldsymbol{x}$, resulting in a higher dimensional vector, $\boldsymbol{p}(\boldsymbol{x})$. For example, if $x_{1}=\left[x_{1} x_{2}\right]$, then its second order polynomial expansion results in the following $6^{\text {th }}$ dimensional vector.

$$
\boldsymbol{p}(\boldsymbol{x})=\left[\begin{array}{llllll}
1 & x_{1} & x_{2} & x_{1}^{2} & x_{2}^{2} & x_{1} x_{2}
\end{array}\right]
$$

Hence, for a sequence of $N: d$-dimensional feature vectors, the $K^{\text {th }}$ order polynomial expansion results in the Nx6 matrix $\boldsymbol{M}$; where,

$$
\boldsymbol{M}=\left[\boldsymbol{p}\left(\boldsymbol{x}_{1}\right) \boldsymbol{p}\left(\boldsymbol{x}_{2}\right) \cdots \boldsymbol{p}\left(\boldsymbol{x}_{N}\right)\right]^{\mathrm{T}}
$$

The polynomial classifier is then trained using the expanded vectors, $\boldsymbol{M}$, to approximate the target using mean squared error as its decisive factor to determine its optimum system parameters (weights), $\boldsymbol{w}$; such as

$$
\boldsymbol{w}=\underset{w}{\arg \min }\left\|\boldsymbol{M w}-\boldsymbol{t}_{x}\right\|_{2}
$$

which results in the well-known solution

$$
\boldsymbol{w}=\left(\boldsymbol{M}^{\mathrm{T}} \boldsymbol{M}\right)^{-1} \boldsymbol{M}^{\mathrm{T}} \boldsymbol{t}_{x}
$$

On testing the trained model, an unknown feature vector, $z$, is expanded to its polynomial terms, $\boldsymbol{p ( z )}$ and by using the previously obtained weights the corresponding output (predicted future price) $\boldsymbol{t}_{\boldsymbol{z}}$, is obtained as follows.

$$
t_{z}=w p(z)
$$

\section{Methodology}

\subsection{Training Scenarios}

In this research, the neural network and polynomial classifiers prediction model are trained on three different training scenarios. These scenarios refer to the number of data points used for training and for validation. In scenario $\# 1,1 / 3$ of the data is used for training and the other $2 / 3$ is used for validation, while in scenario \#2, 1/2 of the data is used for training and the other $1 / 2$ is used for validation. In scenario $\# 3,2 / 3$ of the data is used for training and the remaining $1 / 3$ is used for validation.

The portion of data used for training in the three scenarios consists of data points captured from different periods throughout the available historical data. For example, in scenario \#1, if five hundred data points to be used for training and the remaining 1000 points for validation, then these points will not be taken from one block of the historical data. Instead, several blocks from different periods will form the total five hundred points by taking the first hundred from early data stream, the next two hundred from some middle data, and the last two hundred will be somewhere around most recent data.

This technique is used to assure that both the $\mathrm{NN}$ and the PC prediction methods will learn different market situations and various price patterns in the various market conditions of bear market (period of decline), consolidating market (period of neither growth nor decline), and bull market (period of growth). 


\subsection{Prediction Modes}

Two modes of prediction will be used for predicting stock prices in this study.

Mode 1: Predicting the next day given the five preceding days:

In this mode, the inputs to the model are the closing prices of the previous five trading days, and the desired output (target) is closing price for the following trading day.

$$
\begin{array}{lllll}
d_{1} & \cdots & d_{5} & \rightarrow & d_{6} \\
d_{2} & \cdots & d_{6} & \rightarrow & d_{7} \\
\cdots & & & & \\
d_{n-5} & \cdots & d_{n-1} & \rightarrow & d_{n}
\end{array}
$$

Mode 2: Predicting the next three days given the twelve preceding days:

Similar to the previous mode, however in this mode, the inputs are increased to cover the previous twelve trading days, while the desired output still be the closing prices for the following three trading days.

$$
\begin{array}{ccccccc}
d_{1} & \cdots & d_{1} & \rightarrow & d_{13} & d_{14} & d_{15} \\
d_{2} & \cdots & d_{1} & \rightarrow & d_{14} & d_{15} & d_{16} \\
\cdots & & & & & & \\
d_{n-14} & \cdots & d_{n-3} & \rightarrow & d_{n-2} & d_{n-1} & d_{n}
\end{array}
$$

\subsection{Implementation of Prediction Models}

In this study, two techniques were used for predicting stock prices. The first technique is Neural Networks and the second technique is Polynomial Classifiers. The same inputs are used for both techniques; namely, the historical stocks daily closing prices as explained in Section 4.2. Likewise, the desired outputs (targets) for both techniques are the corresponding future prices according to the prediction modes described in Section 4.2.

The criteria used to measure the prediction accuracy for both the NN and PC is related to the difference between the predicted closing prices and the actual closing prices, as follows.

Let,

$P_{n}=$ Actual closing price for day nine AED

$\hat{P}_{n}^{n}=$ Predicted closing price for day nine $A E D$

$N=$ Number of days in validation

$\varepsilon_{n}=$ prediction error for day nine $A E D$

Then the prediction error for day $n$ is given by

$$
\varepsilon_{n}=P_{n}-\hat{P}_{n}
$$

while the prediction error percentage relative to the actual closing price for day $n$ is given by

$$
\varepsilon_{n} \%=\frac{\varepsilon_{n}}{P_{n}} \times 100 \%
$$

Additionally, the mean absolute error (MAE) in AED over $N$ days is given by

$$
|\bar{\varepsilon}|=\frac{1}{N} \sum_{n=1}^{N}\left|\varepsilon_{n}\right|,
$$

and the mean absolute error percentage (MAEP) is given by

$$
|\bar{\varepsilon} \%|=\frac{1}{N} \sum_{n=1}^{N}\left|\varepsilon_{n} \%\right|
$$

\subsubsection{Neural Network Prediction Technique}

The neural network architecture used here is the multilayer feed forward architecture with one input layer, one hidden layer and one output layer. The training method used here is the standard back propagation method. The network is implemented via MATLAB and the training parameters were set properly to insure convergence for the two prediction modes explained in Section 4.2. For each mode, different ANN models were developed for the three training scenarios as explained in Section 4.3. Consequently, for all the combinations of the three training scenarios and the two prediction modes, 6 different ANNs were generated.

\subsubsection{Polynomial Classifier Prediction Technique}

The inputs and targets used in training the polynomial classifier technique were identical to those used in the neural network technique following the three training scenarios and the two prediction modes. However, for each one of the 6 combinations two PCs were created; one is a $1^{\text {st }}$ order PC and the other is a $2^{\text {nd }}$ order PC. The results obtained from the different ANN and PC combinations are reported in the following section.

\section{Experimental Results and Discussion}

\subsection{Neural Network Prediction Model}

The three training scenarios with two prediction modes were applied to EMAAR and DIB stocks. Following are the results obtained for Mode 1 and Mode 2 for both stocks under study.

\subsubsection{Mode 1: Predicting the Next Day Given the Previous Five Days}

\subsubsection{Emaar Properties (EMAAR)}

Figure 1 shows that ANN achieves a small mean absolute error percentage (MAEP) in all three training scenarios. As expected, the MAEP decreases as the amount of training data is increased. However, the MAEP is still acceptable even with using only $1 / 3$ of the data for training (i.e. training scenario \#1).

It is worth noting here that the distribution of the prediction error (in AED) is found to have a small spread around its average which is close to zero. An example of the distribution of prediction error is depicted in Figure 2 


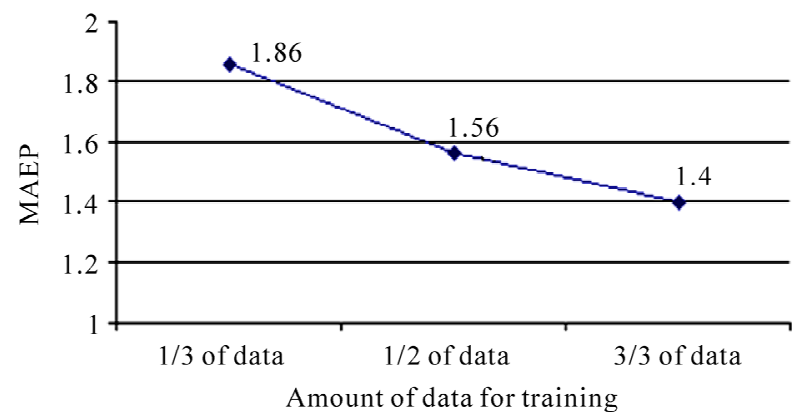

Figure 1. MAEP vs. the amount of training data using ANN.

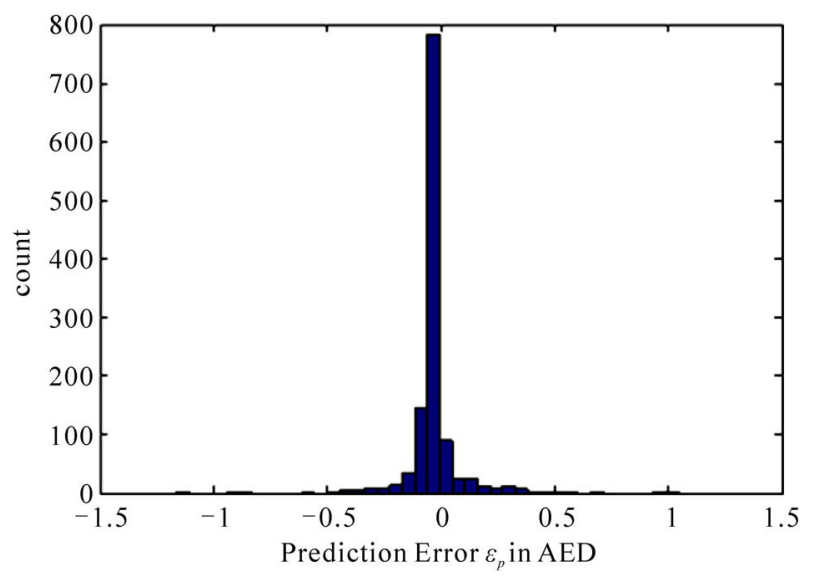

Figure 2. Prediction error histogram; ANN trained on 1/3 of data-Mode 1, Scenario \#1 for EMAAR stock.

which shows the histogram of the prediction error over the entire validation data of scenario \#1.

Further analysis of the histogram of the prediction error in Figure 2 is summarized in Table 1. Table 1 shows that the prediction error percentage $\varepsilon_{n} \%$ is bounded to $\pm 1 \%$ for about one fifth of the validation data. It also shows that $97.13 \%$ of the predicted prices are within $\varepsilon_{n} \%$ $=5 \%$ of the actual prices over the entire validation data. Moreover, $99.92 \%$ of the validation data was predicted within $\varepsilon_{n} \%=10 \%$. Similar analyses were done for the other two training scenarios and the results are summarized in Table 1. Clearly, increasing the training data makes results in a narrower distribution and hence better price prediction.

\subsubsection{Dubai Islamic Bank (DIB)}

Same analyses were done to DIB for the case of prediction Mode 1, and the results are summarized in Table 2. The overall network performance on DIB was superior to its performance on EMAAR especially for the interval from -0.01 to 0.01 where there is a noticeable improvement for all the three training methods.

The improved results shown in Table 2 for DIB could be attributed to the less volatility of DIB stock compared to EMAAR.
Table 1. Intervals of $\varepsilon_{n} \%$ using ANN, prediction Mode 1 for EMAAR stock.

\begin{tabular}{cccc}
\hline \multirow{2}{*}{$\varepsilon_{n} \%$} & \multicolumn{3}{c}{ Percentage of the validation data within the interval } \\
\cline { 2 - 4 } & Scenario \#1 & Scenario \#2 & Scenario \#3 \\
\hline$-1 \%$ to $1 \%$ & $21.52 \%$ & $36.38 \%$ & $49.61 \%$ \\
$-5 \%$ to $5 \%$ & $97.13 \%$ & $97.02 \%$ & $96.85 \%$ \\
$-10 \%$ to $10 \%$ & $99.92 \%$ & $99.68 \%$ & $100.00 \%$ \\
\hline
\end{tabular}

Table 2. Intervals of $\varepsilon_{n} \%$ using ANN, prediction Mode 1 for DIB stock.

\begin{tabular}{cccc}
\hline \multirow{2}{*}{$\varepsilon_{n} \%$} & \multicolumn{3}{c}{ Percentage of the validation data within the interval } \\
\cline { 2 - 4 } & Scenario 1 & Scenario 2 & Scenario 3 \\
\hline$-1 \%$ to $1 \%$ & $58.14 \%$ & $72.59 \%$ & $67.40 \%$ \\
$-5 \%$ to $5 \%$ & $97.01 \%$ & $97.04 \%$ & $96.22 \%$ \\
$10 \%$ to $10 \%$ & $99.35 \%$ & $99.26 \%$ & $99.37 \%$ \\
\hline
\end{tabular}

\subsubsection{Mode 2: Predicting the Next Three Days Given the Previous Twelve Days}

In this mode the number of previous days used for prediction is increased since the number of predicted days is increased to 3 instead of 1 . In this mode, two weeks of historical closing prices plus two days in the third week were used as input to the ANN. This means that we are predicting the last three days of the third week given the previous two weeks and the first two days of the third week. The method was applied to the two stocks under study.

Table 3 shows the results of the ANN for the three training scenarios to predict the 13th, 14th, and 15th day when given the closing prices of the previous 12 days for EMAAR data. As expected, the ANN performs better as the training data increases.

The results obtained in Table $\mathbf{3}$ suggest that increasing the number of days in the historical data beyond 5 does not yield a better prediction in the next day from the previous 12 days (Day 1 ). Moreover, predicting the prices of the 14th and the 15th days (Day 2 and Day 3) yields significantly poorer results than those of predicting Day 1. Similar trends in the prediction results were observed when the NN was applied to DIB stock.

\subsection{Polynomial Classifiers Prediction Model}

The inputs used in the NN model were used for the Polynomial Classifiers model. The model was trained and tested on EMAAR and DIB. First and second order classifiers were examined. Below is a summary of the results of the Polynomial Classifiers compared to the results of 
Neural Network.

\subsubsection{Mode 1: Predicting the Next Day Given the Previous Five Days}

The MAEP results of the 1st order as well as the 2nd order Polynomial Classifiers for predicting the closing price of the 6th day given the closing prices of the previous five days (Mode 1 ) are shown in Table 4 . The table also shows the MAEP for the ANN. The table suggests that the MAEP results for ANN, 1st order PC, and 2nd order PC are comparable and there seems no significant advantage of the PC over ANN. However, it should be noted that PC is far more computationally advantageous over ANN since the training process is done non-iteratively with no concern regarding convergence.

\subsubsection{Mode 2: Predicting the Next Three Days Given the Previous Twelve Days}

In the case of Mode 2 the closing prices of three consecutive days are predicted from the closing prices of the previous 12 days. As shown in Table 5, EMAAR's results indicate that PC yields better results (lower MAEP) than ANN. Once again the MAEP decreases as the training data increase and the MAEP is best for Day1 and worst for Day 3 for all training scenarios.

Unlike EMAAR's results shown in Table 5, the DIB stock results show no advantage of PC over ANN as shown in Table 6.

Table 3. Intervals of $\varepsilon_{n} \%$ using ANN, prediction Mode 2 for EMAAR stock.

\begin{tabular}{cccccccccc}
\hline \multirow{2}{*}{$\varepsilon_{n} \%$ Interval } & \multicolumn{9}{c}{ Percentage of the validation data within the interval } \\
\cline { 2 - 10 } & \multicolumn{3}{c}{ Training Scenario \#1 } & \multicolumn{3}{c}{ Training Scenario \#2 } & \multicolumn{3}{c}{ Training Scenario \#3 } \\
\cline { 2 - 11 } & Day 1 & Day 2 & Day 3 & Day 1 & Day 2 & Day 3 & Day 1 & Day 2 & Day 3 \\
\hline$-1 \%$ to $1 \%$ & $17.61 \%$ & $7.23 \%$ & $5.98 \%$ & $29.63 \%$ & $12.09 \%$ & $7.63 \%$ & $46.10 \%$ & $20.85 \%$ & $15.59 \%$ \\
$-5 \%$ to $5 \%$ & $95.10 \%$ & $58.80 \%$ & $28.41 \%$ & $96.51 \%$ & $83.55 \%$ & $56.86 \%$ & $96.95 \%$ & $88.47 \%$ & $78.47 \%$ \\
$-10 \%$ to $10 \%$ & $99.75 \%$ & $96.84 \%$ & $84.72 \%$ & $99.78 \%$ & $98.04 \%$ & $94.99 \%$ & $99.83 \%$ & $98.31 \%$ & $96.78 \%$ \\
\hline
\end{tabular}

Table 4. Mode 1-MAEP using NN and PC for EMAAR and DIB.

\begin{tabular}{ccccccc}
\hline & \multicolumn{3}{c}{ EMAAR } & & \multicolumn{2}{c}{ DIB } \\
\cline { 2 - 7 } & Scenario \#1 & Scenario \#2 & Scenario \#3 & Scenario \#1 & Scenario \#2 & Scenario \#3 \\
\hline NN & $1.86 \%$ & $1.56 \%$ & $1.40 \%$ & $1.22 \%$ & $1.04 \%$ & $1.18 \%$ \\
$1^{\text {st }}$ order PC & $1.37 \%$ & $1.17 \%$ & $1.28 \%$ & $1.25 \%$ & $1.04 \%$ & $1.18 \%$ \\
$2^{\text {nd }}$ order PC & $1.74 \%$ & $1.32 \%$ & $1.32 \%$ & $1.38 \%$ & $1.33 \%$ & $1.24 \%$ \\
\hline
\end{tabular}

Table 5. Mode 2-MAEP using NN and PC for EMAAR.

\begin{tabular}{|c|c|c|c|c|c|c|c|c|c|}
\hline & \multicolumn{9}{|c|}{ EMAAR } \\
\hline & \multicolumn{3}{|c|}{ Scenario \#1 } & \multicolumn{3}{|c|}{ Scenario \#2 } & \multicolumn{3}{|c|}{ Scenario \#3 } \\
\hline & day 1 & day 2 & day 3 & day 1 & day 2 & day 3 & day 1 & day 2 & day 3 \\
\hline NN & $2.33 \%$ & $4.68 \%$ & $6.73 \%$ & $1.73 \%$ & $3.36 \%$ & $4.81 \%$ & $1.46 \%$ & $2.61 \%$ & $3.58 \%$ \\
\hline $1^{\text {st }}$ order PC & $1.38 \%$ & $2.52 \%$ & $3.32 \%$ & $1.12 \%$ & $2.01 \%$ & $2.79 \%$ & $1.32 \%$ & $2.26 \%$ & $2.91 \%$ \\
\hline $2^{\text {nd }}$ order PC & $1.71 \%$ & $3.45 \%$ & $4.73 \%$ & $1.30 \%$ & $2.42 \%$ & $3.23 \%$ & $1.37 \%$ & $2.17 \%$ & $2.72 \%$ \\
\hline
\end{tabular}

Table 6. Mode 2-MAEP using NN and PC for DIB.

\begin{tabular}{|c|c|c|c|c|c|c|c|c|c|}
\hline & \multicolumn{9}{|c|}{ DIB } \\
\hline & \multicolumn{3}{|c|}{$1 / 3$} & \multicolumn{3}{|c|}{$1 / 2$} & \multicolumn{3}{|c|}{$2 / 3$} \\
\hline & day 1 & day 2 & day 3 & day 1 & day 2 & day 3 & day 1 & day 2 & day 3 \\
\hline NN & $1.28 \%$ & $2.24 \%$ & $3.04 \%$ & $1.05 \%$ & $1.78 \%$ & $2.32 \%$ & $1.19 \%$ & $1.92 \%$ & $3.49 \%$ \\
\hline PC 1st & $1.29 \%$ & $2.42 \%$ & $3.11 \%$ & $1.21 \%$ & $1.82 \%$ & $2.39 \%$ & $1.26 \%$ & $1.94 \%$ & $2.49 \%$ \\
\hline PC 2nd & $1.38 \%$ & $2.51 \%$ & $3.18 \%$ & $1.38 \%$ & $2.44 \%$ & $2.98 \%$ & $1.34 \%$ & $2.15 \%$ & $2.77 \%$ \\
\hline
\end{tabular}




\section{Conclusions}

Two prediction models developed in this study. The first model was developed with the well-known back propagation feed forward neural network. The second model used here is based on polynomial classifiers which are being used for the first time in stock prices prediction. The inputs to both models were identical, and both models were trained and tested on the same data in three different training scenarios and two prediction modes. The data used here is the historical prices for two of the leading stocks in Dubai Financial Market.

In general, both models achieved outstanding results in terms of mean absolute error percentage (MEAP). Both models achieved around 1.5\% MEAP in predicting the next day, 2.5\% MEAP in predicting the second day, and around 4\% MEAP in predicting the third day. The prediction accuracy of the two models was certainly remarkable, where around $60 \%$ of the predicted prices of the first day, $50 \%$ of the predicted prices of the second day, and $35 \%$ of the predicted prices of the third day, were all within $-1 \%$ to $1 \%$ of the actual prices of the three days.

When comparing the neural network and polynomial classifiers prediction models, it was found that first order polynomial classifier performed comparable to or slightly better than the neural network. Whereas the second order polynomial classifier could barely achieve similar results on the stocks used in this study. Further work can be done using other stocks in similar emerging markets and mature markets, to verify this conclusion.

On the other hand it should be noted that PC is a lot more computationally efficient than ANN since its weights can be obtained directly and non-iteratively from a closed formula as shown in Section 3.1.

\section{REFERENCES}

[1] E. Fama, "Efficient Capital Markets: A Review of Theory and Empirical Work,” Journal of Finance, Vol. 25, No. 2, 1970, pp. 383-417. doi:10.2307/2325486

[2] J. Yao, C. Tan and H. Poh, "Neural Networks for Technical Analysis: A Study on KLCI," International Journal of Theoretical and Applied Finance, Vol. 2, No. 2, 1999, pp. 221-241. doi:10.1142/S0219024999000145

[3] D. Brownstone, "Using Percentage Accuracy to Measure Neural Network Predictions in Stock Market Movements," Neurocomputing, Vol. 10, No. 3, 1996, pp. 237250. doi:10.1016/0925-2312(95)00052-6

[4] K. H. Lee and G. S. Jo, "Expert System for Predicting Stock Market Timing Using a Candlestick Chart,” Expert Systems with Applications, Vol. 16, No. 4, 1999, pp. 357364. doi:10.1016/S0957-4174(99)00011-1

[5] W. Leigh, M. Paz and R. Purvis, "An Anaysis of a Hybrid Neural Network and Pattern Recognition Technique for
Predicting Short-Term Increases in the NYSE Composite Index,” Omega, Vol. 30, No. 2, 2002, pp. 69-76. doi:10.1016/S0305-0483(01)00057-3

[6] R. Choudhry and K. Garg, "A Hybrid Machine Learning System for Stock Market Forecasting,” World Academy of Science, Engineering and Technology, Vol. 39, 2008, pp. 315-318.

[7] G. Armano, M. Marchesi and A. Murru, "A Hqybrid Genetic-Neural Architecture for Stock Indexes Forecasting," Information Sciences, Vol. 170, No. 1, 2005, pp. 3-33. doi:10.1016/j.ins.2003.03.023

[8] J. Yao and C. Tan, "A Case Study on Using Neural Networks to Perform Technical Forecasting of Forex," Neurocomputing, Vol. 34, No. 1-4, 2000, pp. 79-98. doi:10.1016/S0925-2312(00)00300-3

[9] D. Senol and M. Oztuman, "Stock Price Direction Prediction Using Artificial Neural Network Approach: The Case of Turkey," Journal of Artificial Intelligence, Vol. 1, No. 2, 2008, pp. 70-77. doi:10.3923/jai.2008.70.77

[10] A.-S. Chen, M. Leung and H. Daouk, “Application of Neural Networks to an Emerging Financial Market: Forecasting and Trading the Taiwan Stock Index," Computers \& Operations Research, Vol. 30, No. 6, 2003, pp. 901-923. doi:10.1016/S0305-0548(02)00037-0

[11] D. Enke and S. Thawornwong, "The Use of Data Mining and Neural Networks for Forecasting Stock Market Returns,” Expert Systems with Applications, Vol. 29, No. 4, 2005, pp. 927-940. doi:10.1016/j.eswa.2005.06.024

[12] Q. Cao, K. Leggio and M. Schniederjans, “A Comparison between Fama and French's Model and Artificial Neural Networks in Predicting the Chinese Stock Market," Computers \& Operations Research, Vol. 32, No. 10, 2005, pp. 2499-2512. doi:10.1016/j.cor.2004.03.015

[13] I. Kaastra and M. Boyd, "Designing a Neural Network for Forecasting Financial and Economic Time Series,” Neurocomputing, Vol. 10, No. 3, 1996, pp. 215-236. doi:10.1016/0925-2312(95)00039-9

[14] N. Kohzadi, M. Boyd, B. Kermanshahi and I. A. Kaastra, "Comparison of Artificial Neural Network and Time Series Models for Forecasting Commodity Prices," Neurocomputting, Vol. 10, No. 2, 1996, pp. 169-181. doi:10.1016/0925-2312(95)00020-8

[15] D. Olsona and C. Mossmanb, "Neural Network Forecasts of Canadian Stock Returns Using Accounting Ratios," International Journal of Forecasting, Vol. 19, No. 3, 2003, pp. 453-465. doi:10.1016/S0169-2070(02)00058-4

[16] K. Assaleh and M. Al-Rousan, "Recognition of Arabic Sign Language Alphabet Using Polynomial Classifiers," EURASIP Journal of Applied Signal Processing, Vol. 2005, No. 13, 2005, pp. 2136-2145. doi:10.1155/ASP.2005.2136

[17] K. T. Assaleh and W. M. Campbell, "Speaker Identification using a Polynomial-Based Classifier," Fifth International Symposium on Signal Processing and Its Applications ISSPA, Brisbane, Vol. 1, August 1999, pp. 115118.

[18] W. M. Campbell, K. T. Assaleh and C. C. Broun, “Speaker 
Recognition with Polynomial Classifiers,” IEEE Transactions on Speech and Audio Processing, Vol. 10, No. 4, May 2002, pp. 205-212. doi:10.1109/TSA.2002.1011533

[19] C.-L. Liu and H. Sako, “Class-Specific Feature Polynomial Classifier for Pattern Classification and Its Application to Handwritten Numeral Recognition," Pattern Recognition, Vol. 39, No. 4, 2006, pp. 669-681. doi:10.1016/j.patcog.2005.04.021

[20] I. Deiab, K. Assaleh and F. Hammad, “On Modeling of
Tool Wear Using Sensor Fusion and Polynomial Classifiers,” Mechanical Systems and Signal Processing, Vol. 23, No. 5, July 2009, pp. 1719-1729. doi:10.1016/j.ymssp.2009.02.001

[21] K. Assaleh and H. Al-Nashash, "A Novel Technique for the Extraction of Fetal ECG Using Polynomial Networks," IEEE Transactions on Biomedical Engineering, Vol. 52, No. 6, June 2005, pp. 1148-1152. doi:10.1109/TBME.2005.844046 